\title{
Research on Avoid Repetition Information Flooding Broadcasting Protocol
}

\author{
Lixia Liu and Yiqi Zhuang, Member, IEEE
}

\begin{abstract}
Distributed ad hoc broadcasting is not only the foundation for unicast, multicast as well as deterministic broadcast routing protocols to detect and maintain their routing, but itself can be used as approach to transmit messages for unicast, multicast and broadcast communication. In order to avoid the problems of poor broadcast reliability, large transmission delay and more network resource occupation that caused by broadcast storms, adjacent conflict, hidden collisions in the packet radio network application of traditional broadcasting protocol, the paper presented an Avoid Repetition Information Flooding Broadcasting Protocol (ARIFBP) and mainly researched on its material algorithm and related key problems for practical applications based on Collision Avoid Multiple Access (CAMA) protocol. The proposition of ARIFBP is of great significance for the improvement of routing protocols and researches on ad hoc packet radio network.
\end{abstract}

Index Terms-packet radio network, broadcast, flooding, avoid repetition information

\section{INTRODUCTION}

In the networking process of multi-hop packet radio network, distributed ad hoc broadcasting is a necessary operation, which is not only the foundation for unicast, multicast as well as deterministic broadcast routing protocols to detect and maintain their routing, but it can be used as means to transmit unicast, multicast and broadcast messages. Flooding is a king of ad hoc technology that has been widely used in the practical network applications. But when applied in multi-hop packet radio network, it will introduce the problems of broadcast storms, adjacent conflict, hidden collisions and etc, which will result in bad broadcast reliability, larger transmission delay and more network resource occupation. Aim at the characteristics of multi-hop packet radio network, the paper brought out the ARIFBP. It utilizes CAMA firstly to solve the problems of adjacent conflict and hidden collisions, which is a kind of non-collision and high efficiency preferential RTS/CTS appointment multiple access mechanism that can not only solve problems of adjacent conflict and hidden collisions, but also effectively support QoS of multimedia traffic and insure prior traffic can be accessed in priority. At the same time, the

Manuscript received October 19, 2009. This work was supported in part by National Natural Science Foundation of China (Grant No.60276028) and the National Defense Pre-Research Foundation of China (Grant No. 51411040601DZ014).

Lixia Liu is with Microelectronics Institute, Xidian University and the Department of Communication Engineering, Engineering College of Armed Police Force (email: wjllx939@tom.com). Yiqi Zhuang is with Microelectronics Institute, Xidian University (email: zhuangyiqi@126.com).
ARIFBP makes use of local neighbor information and collected repetition broadcasting information to avoid broadcast storms, so that the repeated broadcasting information of flooding can be effectively avoid, and improve the broadcast transmission speed and network throughput, which has good reliability, too. The arrangement of the paper is as following: Section 2 analyzes the related research works of broadcasting protocol and points out their advantages and disadvantages; In section 3, the detail algorithm of the paper proposed ARIFBP; Section 4 researches key problems of forward delay, adjacent information acquisition, one-way link and data structure that relates to practical applications of ARIFBP; Section 5 concludes our work.

\section{RELATED RESEARCH WORKS OF FLOODING BROADCASTING PROTOCOL}

The broadcasting in multi-hop packet radio network can be divided into two types of local broadcasting and global broadcasting. Local broadcasting indicates that a node only needs to send a message that can be transmitted to all the communication nodes that within its wireless transmitter scope, which is the difference from wire network. Global broadcasting indicates that the message that sent by source node can be transmitted to all the accessible nodes except for the source node after multi hops, which is also be called one to all point communication. According to the application requirements, the broadcasting can also be divided into service types of basic broadcasting, reliable broadcasting, real-time broadcasting and etc (If it is not stated specially, the broadcasting refers to basic global broadcasting in this paper). In accordance with the different function of node in the broadcasting process, the network nodes can be divided into re-broadcasting node and leaf node. For the re-broadcasting node, when receiving a non-repeat broadcasting message, it will transmit the message to all adjacent nodes by local broadcasting. As regard to leaf node, it only receives broadcasting message but not rebroadcast it. Seeing the re-broadcasting node as the dominating node, all the re-broadcasting nodes can constitute the Connected Dominating Set of network, so the broadcasting problem can be transformed into the Minimum Connected Dominating Set problem of Graph Theory.

At present, the broadcasting algorithm can be divided into two kinds of centralized algorithm and distributed algorithm. The centralized algorithm solves the problem assuming that through some way the comprehensive information of the network can be obtained, which can achieve better 
Connected Dominating Set [2]. The centralized algorithm was usually applied in wireless network whose topology structure is fixed or changes slowly, as well as the circumstance that rapidly network topology update to adapt changes of mobile ad hoc network structure, which instead induces the problem that how the topology update information effectively broadcast. So for the mobile ad hoc network, it must look for a distributed algorithm that can effectively broadcast merely depend on local network topology information. To solve the problem, researchers have brought out many kinds of distributed algorithms [3, 4]. In general, the MCDS problem in Graph Theory in fact is NP problem. Although these algorithms have strong distribution and can achieve most approximate solution, but the reliability was not so strong as to be applied in practice. To solve the problem, the engineers has brought out mechanism to reduce broadcast overhead by use of position information $[5,6]$ or available path information [7], the most advantage of which is that the spatial localization of node mobile was utilized. It is benefit for broadcasting inquiring near the original paths and executing deterministic routing communication, but it is not suitable for general basic broadcasting communication. In addition, Ref. 8 also proposed many ways to reduce the redundant broadcast information, such as count-based method and probabilistic method. These two methods have the advantage of simple, but there are blind. Because when the node executes re-broadcasting determination, it has not really considered the existing connection condition of the network. So even under the circumstance of non-conflict and non-mistake, some nodes still can not receive the broadcasting message from the source node.

At present, in the actual applications of multi-hop packet radio network, flooding is the basic approach to achieve broadcasting. In this way, each node is re-broadcasting node, when the source node can be seen as the first re-broadcasting node. When each node receives a broadcasting message for the first time, which will be simply sends to all the adjacent nodes after a period of delay. The most advantage of flooding is simple, non-state and high reliability. But the shortcoming of flooding is also obvious, namely great redundancy making the network throughput significantly decreased, and the potential problem of broadcasting storm [8], which may cause a lot of conflict and collision in the wireless network and decrease the reliability. Here we will briefly present the results of analysis:

1) Redundant Rebroadcasts. Analysis showed that when the node $u$ receives a broadcast message from its neighbor $v$ and forwards the message, the forwarding node $u$ can cover $41 \%$ of operating the new area on average. If there is $k$-neighbors forward the implementation of the operation, when $k \geq 4$, the forward operating area to cover the expected value of the new area less than $0.05 \%$.

2) Contention. When a node to broadcast a message, if the adjacent node must also be simultaneously broadcast, then they compete using wireless transmission channel, which will cause conflict. The more are dense the network nodes, the greater is the likelihood of conflict. The simulation results show that when the neighbor number $N \geq 6$, all the neighbor nodes the collision probability over $80 \%$.

3) Collision. Because of the hidden terminal problem, when $\mathrm{C}$ is the neighbor of node $A, B$ but $A$ and $B$ are not neighbors, if $A$ and $B$ simultaneously broadcast operation, the broadcast message in node $C$ will suffer from collision lost.

Through the analysis of the broadcasting problems above, we can see that the basic approach to achieve broadcasting in the mobile ad hoc network currently is flooding. But the flooding broadcast has great redundancy, which has advantages and disadvantages. In one aspect, the redundancy itself will improve the receive efficiency of broadcasting messages. But in another aspect, the redundancy will increase the conflict that caused by channel sharing and collision, which will decreases the receive rate of messages and waste wireless link bandwidth, node processing time and power energy. So for the design of new broadcasting algorithm, we should achieve reasonable compromise between the overhead of broadcasting and reliability and adopt less conflict and collision channel multiple access protocol. Aim at this, based on the CAMA protocol, the paper brings out an ARIFBP, which makes use of the collection of adjacent repeated broadcasting information and local neighboring information to effectively avoid the repetition information of flooding, thus to improve the broadcasting transmission speed and network throughput. The ARIFBP has good reliability.

\section{ARIFBP ALGORITHM}

\section{A. Network Model}

In order to facilitate APIFBP research, we make the following assumptions of network model:

1) All the mobile nodes adopt omni-directional antenna.

2) The wireless channel is shared by network nodes. The MAC protocol adopts the above mentioned CAMA protocol.

3) The message that sent by one node can be transmitted to all the communication nodes within its wireless transmitter scope.

4) The network is of flat structure, which means all nodes in the network are independent and equal, so there is no central control node of base station or cluster head. The network is of distributed operation.

5) By use of some mechanism, such as periodical or event driven mechanism, each node can exchange neighboring set information with all the adjacent nodes to obtain the local network topology information within two hops.

\section{B. ARIFBP Algorithm Description}

To convenient the description of ARIFBP algorithm, the following expressions can be regulated firstly according to the Graph Theory:

$N(u)$ is the neighboring collect of the node $u . C(u, m)$ is used to express the broadcasting coverage collect of the message $m$ from node $u$. If a node is included in the broadcasting coverage collect, we can think that the node has received broadcasting message. 
Assume the distance of each side is 1 and $D(u, v)$ is the shortest path between $u$ and $v$. The algorithm of ARIFBP can be described as following:

1) For the source node $s$, it will send the message $m$ to all its adjacent nodes by local broadcasting and then discard the received repetition message $m$ afterwards.

2) For other nodes, such as node $u$, when it receives a broadcasting message $m$, if the transmitter node is node $t$, the node $u$ will execute the following operation: If $N(u)$ $\subseteq N(t) \cup\{t\}$, do not rebroadcast the message and discard later received repeated message $m$. Otherwise, if it is the first time for the message $m$ to be received, set $C(u, m)=N(t) \cup\{t\}$ and delay the rebroadcast operation of message $m$ for a period. In the delay period, if repeated message $m$ is received, discard it and record the node information that received messages in the broadcasting coverage collection, and then set $C(u, m)$ $=C(u, m) \cup N(t) \cup\{t\}$. After the delay period is finished, if $N(u) \subseteq C(u, m)$, do not need to rebroadcast the message. Otherwise, rebroadcast the message $m$ by local broadcasting and discard later received repetition message $m$.

\section{Correctness Prove of ARIFBP Algorithm}

To ensure to the correctness of the above mentioned ARIFBP algorithm, we can prove the following theorem: Assume the transmission link is non-error and there is no loss that caused by collision in the message transmission process, for a given connected undirected graph $G=(V, E)$, in the above algorithm, any node except for the source node will at least receive one copy of the broadcasting message.

To prove the above theorem, we can assume that $s$ is the broadcasting source node and $m$ is the message that be sent by $s$. First of all, all adjacent nodes of node $s$ will at least receive one copy of $m$. Then we suppose that the result is correct for all the nodes whose distance to node $s$ is $k$ hops.

Suppose the distance from node $v$ to node $s$ is $k+1$ hops and the node $w$ is the former one node along the shortest path $D(v, s)$ from $v$ to $s$. Obviously, the distance from node $w$ to node $s$ is $k$ hops. According to the supposition, the node $w$ will also receive one copy of message $m$. If the node $w$ rebroadcast the message $m$, the node $v$ will receive one copy of $m$, the conclusion was established. Otherwise, the neighboring set of $w$ must be included in the broadcasting coverage set $C(w, m)$, so $v \in C(w, m)$. Noted that $C(w, m)$ is the node set that be covered by the former message transmission, so the node $\mathrm{v}$ will receive one copy of message $m$ from the former transmission. We can conclude by the induction that the result is correct.

\section{Key Problems of ARIFBP Algorithm}

\section{A. Problem of Forwarding Delay}

The forwarding delay time has an important impact on the performance of ARIFBP. In general, under the circumstance of channel sharing and can be accessed randomly, in order to reduce the collision that caused by simultaneous transmission, the forwarding of broadcasting message will delay a long period of random time. Although in the delay period, the re-broadcasting node can also avoid repeated same broadcasting through receiving the re-broadcasting from adjacent nodes. But in fact, for the many re-broadcasting nodes that have same receiving adjacent nodes, the broadcasting from any rep-broadcasting node can make other re-broadcasting node avoid redundant broadcasting. But for the re-broadcasting nodes that have different receiving adjacent nodes, because they must rebroadcast to its own different adjacent nodes, there is no way to prevent the re-broadcasting operation of other re-broadcasting nodes. Therefore, the general longer random delay time can not reduce redundant broadcasting and can only be used to reduce adjacent conflict and hidden collision, which will increase the broadcasting delay and make the local network topology on which the algorithm depends not be updated timely, so it will further lead to decrease the reliability of broadcasting.

The ARIFBP depended channel multiple access protocol is the above mentioned CAMA. When, the forward delay time may be same as the determinate network access delay (NAD) of CAMA, the value of which is usually in the scope from 0 to $N$ small time slot $n \Delta t$. The average delay is $(N \times \Delta t) / 2$, where $N$ is the node number of network. The CAMA based ARIFBP can not only avoid the $80 \%$ adjacent conflict problem, hidden collision problem and broadcasting storm problem of redundant re-broadcasting of [8], but also can effectively reduce the broadcasting transmission delay and improve the reliability of broadcasting transmission.

\section{B. Problem of One-way Link}

In the wireless network, for the difference of actual power, receive sensitivity, located terrain and etc, the one-way problem may be caused. If the practical application problem has noe been considered by ARIFBP, the one-way link will lead to error when the node determine re-broadcasting operation according to broadcasting coverage set, which will lead to some nodes can not receive broadcast messages.

For example, assume that the link $A B$ in Fig. 1 is a one-way link. $B$ can receive the Hello messages and broadcasting messages from $A$, but $A$ cannot receive these from $B$. Suppose that $B$ receive a broadcasting message from $C$ and rebroadcast it to $D$. For the node $D$, because the neighboring table of $B$ is $\{A, D, C\}$, so the broadcasting coverage set of $D$ is $\{A, D, C, B\}$. As the neighbors of $D$ are all be included in the broadcasting coverage set, so $D$ will not rebroadcast message, which will eventually leads to $A$ can not receive broadcasting messages from $C$.

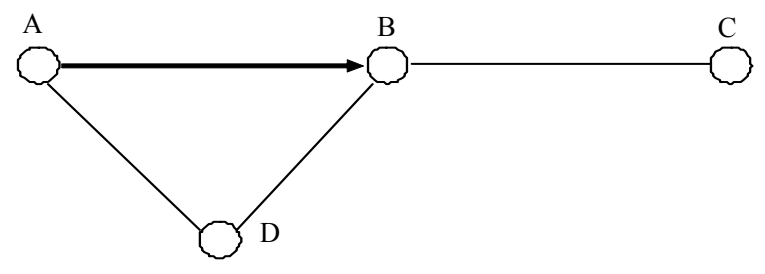

Figure 1. The sketch map of one-way link.

In order to reduce the affect that caused by one-way link, the node need to know whether some link is one-way link. For that, we must improve the ARIFBP and determine the 
following rules:

Rule 1: The Hello message includes determinate link directional information.

Rule 2: If $A$ to $B$ as a one-way link, when $B$ adds its adjacent nodes into the broadcasting coverage set, the adjacent node $A$ along the one-way link can not be considered.

Considering that node $A$ may just enter into the wireless coverage scope for not a long time, the actual link also exists, but for the Min_Period cause, the adjacent relation has not been established. At this moment, to enhance the reliability of broadcasting and establishment of timely adjacent relationship, the following rules can be introduced:

Rule 3: If $A$ is not in the neighboring table of $B$, when the node $B$ receives broadcasting message from $A, B$ should rebroadcast the broadcasting message of $A$.

\section{Problem of Adjacent Information Acquisition}

In general, the ARIFBP can utilize Hello process to obtain the adjacent node information within two hops. But in practical realization, the Hello process can be integrated with other network layer protocols. Such as in the distance vector protocol, the Hello process of ARIFBP can be cancelled, but to obtain adjacent information directly from the neighboring routing table. At the same time, the routing protocol can utilize ARIFBP to improve the broadcasting mechanism of routing finding and maintenance, so that improve network performance. In addition, the local topology information that obtained in the Hello process or direct ARIFBP broadcasting can also be used by routing protocol. For example, in the on-demand routing protocol, the unnecessary routing finding operation can be reduced. Therefore, the ARIFBP can be the important basis for the improvement of routing protocols.

The ARIFBP use periodical and event-driven mechanism to send Hello message. The event-driven mechanism means that the Hello process is triggered under the circumstance of finding adjacent node change or the node itself receiving abnormal packet. The periodical driven mechanism indicates that after each Min_Period, the Hello process will be triggered. The Min_Period determines the basic update frequency of adjacent information, but Max_Period determines the response tardy degree of protocol to disabled adjacent information. The value of Max_Period mainly depends on the value of Min_Period. Less the value of Min_Period, the more real is adjacent information that the node obtained and more reliability. However, too frequent Hello process will generate large amount of Hello message in the network, accordingly occupy large amount of network bandwidth and increase the protocol overhead, so the performance of protocol will be decreased. Therefore, the value of Min_Period should be considered compromised. The reasonable value should meet the following requirement: update frequency is slightly faster than the changes frequency of link.

\section{Problem of Data Structure}

The ARIFBP is same as other flooding protocol. Each node needs to maintain a repeated message table to be used for repetition message detection. But for each item in the repeated message table of ARIFBP, it needs to add a broadcasting coverage set region to record information of redundant broadcasting message and provide the basis for node to determine whether rebroadcast message.

In the ARIFBP, by exchanging Hello message with adjacent nodes, each node should also maintain a two-hop adjacent table, which records the node's adjacent link state information, adjacent node's adjacent link state information as well as one-way or two-way link indication included link state, all these are used for the determination of ARIFBP re-broadcasting message.

\section{E. Application Problem of Hierarchy Structure Network}

In ordr to facilitate the discussion, the network of ARIFBP is supposed to be flat structure, which is more suitable for small scale wireless network. In fact, for the larger scale network, router-based partition or cluster-based hierarchical network structure is usually adopted. In general, the router-based subnets utilize ARIFBP to broadcast. While the cluster-head and cluster member based broadcasting can use simple local broadcasting. The network between routers or clusters can be seen as a logical core subnet. At this moment, the routers can utilize multiple local broadcasting or another channel local broadcasting among clusters to directly execute global broadcast in the logical core subnets by ARIFBP.

\section{CONCLUSION}

The paper focuses on the basic distributed ad hoc broadcasting problem of multi-hop packet radio network, which is not only the foundation for unicast, multicast as well as deterministic broadcast routing protocols to detect and maintain their routing, but it can be used as means to transmit unicast, multicast and broadcast messages. Flooding is the widely used broadcast technology in practical applications, but for the multi-hop topology structure of packet radio network and possible frequent changes, it will brings out problems of broadcasting storms, adjacent conflict and hidden collision in the applications, which will lead to bad broadcast reliability, larger transmission delay and more network resource occupation. To solve these problems, the paper brought out an ARIFBP, the basic idea of which is to makes use of the collection of adjacent repeated broadcasting information and local neighboring information to effectively avoid the repetition information of flooding, thus to improve the broadcasting transmission speed and network throughput. The paper also proved the correctness of ARIFBP algorithm and researched the problems of forwarding delay, adjacent information acquisition, one-way link, data structure and etc. The proposition of ARIFBP is of great basic functions for ad hoc of distributed multiple packet radio network and mobile interconnection of hierarchical and distributed mobile wireless network.

\section{REFERENCES}

[1] Sudipto Guha and Samir Khuller. Approximation Algorithms for Connected Dominating Sets. The Fourth Annual European Symposium on Algorithms, Barcelona, Spain, Sept. 1996, pp. 374-387.

[2] Bevan Das and V. Bharghavan. Routing in Ad-Hoc Networks Using Minimum Connected Dominating Sets. IEEE International Conference on Communications (ICC'97), Montreal, Canada, June 1997, pp. 376--380. 
[3] Ben Liang, Zygmunt J. Haas. Hybrid Routing in Ad Hoc Network with a Dynamic Virtual Backbone. IEEE Transactions on Wireless Communications, 2006, vol 5, pp. 1-14.

[4] Jie Wu and Hailan Lt. On Calculating Connected Dominating Set for Efficient Routing in Ad Hoc Wireless Networks. The Third International Workshop on Discrete Algorithms and Methods for Mobile Computing and Communications (DIALM'99), Seattle, WA, August 1999, pp. 7-14.

[5] Young-Bae Ko and Nitin H. Vaidya. Location-Aided Routing (LAR) in Mobile Ad Hoc Networks. The Fourth Annual ACM/IEEE International Conference on Mobile Computing and Networking (MOBICOM'98), Dallas, Texas, October 1998, pp. 307-321.

[6] S. Basagni, I. Chlamtac, and V. R. Syrotiuk. Dynamic Source Routing for Ad Hoc Networks Using the Global Positioning System. The IEEE Wireless Communications and Networking Conference 1999 (WCNC'99), New Orleans, LA, September 1999, vol. 1, pp. 301-305..

[7] R. Castaneda, and Samir R. Das. Query Localization Techniques for On-demand Routing Protocols in Ad Hoc Networks. The Filth Annual ACM/IEEE International Conference on Mobile Computing and Networking (MOBICOM'99), Seattle, WA, August 1999, pp. 186-194.

[8] Sze-Yao Ni, Yu-Chee Tseng, Y. S. Chen and J. P. Sheu. The Broadcast Storm Problem in a Mobile Ad Hoc Network. The Fifth Annual ACM/IEEE International Conference on Mobile Computing and Networking (MOBICOM'99), Seattle, WA, August 1999, pp. 151-162.

First A. Author: Lixia Liu was born in Taiyuan, Shanxi Province, China, in 1975. She received the B.S. degree in computer applications from Armed Police Technology Academy, Xian, China, in 1998 and M.S. degree in computer application technologies from Northwestern Polytechnical University, Xian, China, in 2003. She is now a D.S. student in Xidian University, Xian, China. Her research interests include Bluetooth communication system chip designing, embedded system developing and integrating.

E-mail: wjllx939@tom.com

B. Author: Yiqi Zhuang was born in Xian, China, in 1957. He received the B.S., M. S. and PhD degree from Xidian University in 1982, 1986 and 1995 respectively. He is the president of the Microelectronics Institute and advisor for doctoral students in Xidian University now. In recent years, his research interests focus on designing wireless communication and information appliances core chip, detecting weak signal, developing virtual apparatus, and researching microelectronic devices noise and reliability. He has more than 100 journal and conference publications in subject of his interests.

E-mail: zhuangyiqi@126.com 